Portland State University

PDXScholar

\title{
High-velocity Limits for the Ratio of Double to Single Ionisation of Helium by Projectiles with Electrons
}

\author{
Y. D. Wang \\ Jack C. Straton \\ Portland State University, straton@pdx.edu \\ James H. McGuire \\ R. D. DuBois
}

Follow this and additional works at: https://pdxscholar.library.pdx.edu/phy_fac

Part of the Atomic, Molecular and Optical Physics Commons

Let us know how access to this document benefits you.

\section{Citation Details}

Wang, Y. D., Straton, J. C., McGuire, J. H., \& DuBois, R. D. (1990). High-velocity limits for the ratio of double to single ionisation of helium by projectiles with electrons. Journal of Physics B: Atomic, Molecular and Optical Physics, 23(9), L133.

This Letter to the Editor is brought to you for free and open access. It has been accepted for inclusion in Physics Faculty Publications and Presentations by an authorized administrator of PDXScholar. Please contact us if we can make this document more accessible: pdxscholar@pdx.edu. 


\section{High-velocity limits for the ratio of double to single ionisation of helium by projectiles with electrons}

To cite this article: Y D Wang et al 1990 J. Phys. B: At. Mol. Opt. Phys. 23 L133

\section{Recent citations}

- The ratio of cross sections for double to
single ionization of helium by high energy
photons and charged particles
$\mathrm{JH}$ McGuire et al
- Theory of projectile ionization by molecular
hydrogen targets

Wang, Y. D. et al

View the article online for updates and enhancements.

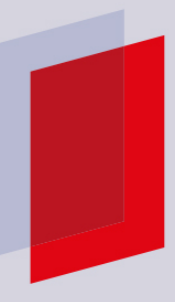

\section{IOP ebooks'}

Bringing you innovative digital publishing with leading voices

to create your essential collection of books in STEM research.

Start exploring the collection - download the first chapter of every title for free. 


\title{
LETTER TO THE EDITOR
}

\section{High-velocity limits for the ratio of double to single ionisation of helium by projectiles with electrons}

\author{
Y D Wang $\nmid$, Jack C Straton $\dagger$, J H McGuire $\nmid$ and R D DuBois $\ddagger$ \\ $\dagger$ J R McDonald Laboratory, Department of Physics, Kansas State University, Manhattan, \\ KS 66506, USA \\ $\doteqdot$ Pacific Northwest Laboratory, Richland, WA 99352, USA
}

Received 26 September 1989, in final form 21 February 1990

\begin{abstract}
The ratio of total double to total single ionisation cross sections, $\mathscr{R}$, by high velocity projectiles containing electrons is examined in the first Born approximation. For ionisation of helium by $\mathrm{H}^{+}, \mathrm{H}^{0}$ and $\mathrm{He}^{0}$ impact, it is found that $\mathscr{R}_{\mathrm{H}^{+}}<\mathscr{R}_{\mathrm{H}^{0}}<\mathscr{R}_{\mathrm{He}^{0}}$. Connection with Bohr's free-collision model for collisions with projectile electrons is given.
\end{abstract}

At high projectile velocities much of the physics of single and multiple ionisation can be understood within the framework of a first Born approximation (Bates and Griffing 1955, Bell et al 1969, Inokuti 1971, Manson et al 1975, McGuire 1984, 1987). For a bare projectile, observed cross sections for both single and double ionisation vary as $Z^{2}$, where $Z$ is the charge of projectile, in accord with the first Born approximation (Haugen et al 1982). For a projectile with bound electrons, it has been shown for single ionisation (Briggs and Taulbjerg 1978, Inokuti 1979, McGuire et al 1981, Anholt $1986)$ that $Z^{2}$ is replaced by $Z_{\text {eff }}^{2}(Q)$ which describes the screening and antiscreening of the projectile nucleus by the electrons. (Here $Q$ is the momentum transfer.) Thus the single ionisation cross sections for bare projectiles and projectiles with electrons can be interrelated through the effective charges. Given the first Born inter-relationship for single ionisation for bare and charged projectiles, it is possible to begin to consider the effect of projectile electrons on the ratio of double to single ionisation at high collision velocities. In this letter the ratios between both differential double to single ionisation cross sections $R$ and total double to single ionisation cross sections $\mathscr{R}$ are examined. A first-order interrelation of $\mathscr{R}$ for projectiles with and without electrons is given.

At high projectile velocities, $v_{\mathrm{p}}$, it has been shown (Mittleman 1966, Byron and Joachain 1967, Carlson 1967, Åberg 1973, Carlson and Nextor 1973, Quarles 1973, Carter and Kelly 1981, McGuire 1984, 1987) that the total double ionisation cross section $\left(\sigma^{++}\right)$is related to the total single ionisation cross section $\left(\sigma^{+}\right)$by

$$
\mathscr{R}=\lim _{v_{\mathrm{p}} \rightarrow \infty} \sigma^{++} / \sigma^{+} \rightarrow \text { constant. }
$$

The ratio of double to single ionisation is sensitive to electron correlation.

Let us now consider the differential single ionisation cross section, $\mathrm{d} \sigma^{+}$, and differential double ionisation cross section, $\mathrm{d} \sigma^{++}$. By integrating over one of the ejected 
electron momenta, e.g. the slower electron, one may write, in accord with equation (1)

$$
\frac{\mathrm{d} \sigma^{++}}{\mathrm{d} Q \mathrm{~d} \varepsilon}=R(Q, \varepsilon) \frac{\mathrm{d} \sigma^{+}}{\mathrm{d} Q \mathrm{~d} \varepsilon} \sim Z^{2} \quad \text { or } \quad Z_{\mathrm{eff}}^{2}(Q)
$$

where $\varepsilon$ is the energy of the other outgoing electron. Here the $Z^{2}$ or $Z_{\text {eff }}^{2}(Q)$ dependence comes from the first Born approximation for single ionisation due to bare projectiles or projectiles with electrons. The proportionality factor $R$ can be taken as a ratio between differential double and single ionisation cross sections. Generally $R$ depends on both $Q$ and $\varepsilon$.

A relation between $R$ and $\mathscr{R}$ can be obtained from equations (1) and (2) for bare projectiles and for projectiles with electrons. First, for bare projectiles (such as $\mathrm{e}^{-}$, $\left.\mathrm{H}^{+}, \mathrm{He}^{2+}, e t c\right)$, we have

$$
\begin{aligned}
\mathscr{R} & =\int \mathrm{d} \varepsilon \int R(Q, \varepsilon) \frac{\mathrm{d} \sigma^{+}}{\mathrm{d} \varepsilon \mathrm{d} Q} \mathrm{~d} Q\left(\int \mathrm{d} \varepsilon \int \frac{\mathrm{d} \sigma^{+}}{\mathrm{d} \varepsilon \mathrm{d} Q} \mathrm{~d} Q\right)^{-1} \\
& =\int \mathrm{d} \varepsilon \int R(Q, \varepsilon) \frac{\mathrm{d} \sigma^{+}}{\mathrm{d} \varepsilon \mathrm{d} Q} \mathrm{~d} Q\left(\sigma^{+}\right)^{-1} .
\end{aligned}
$$

The minimum value of $Q$ depends on the ionisation potential, $I$, which is different for single and double ionisation. For fast heavy projectiles, $\varepsilon$ ranges from threshold to infinity.

For projectiles carrying $N$ electrons (such as $\mathrm{H}^{0}, \mathrm{He}^{0}$, etc), in the first Born approximation, the single ionisation differential cross section $\left(\mathrm{d} \sigma^{+} \equiv \mathrm{d} \sigma_{\mathrm{H}^{0}}^{+}, \mathrm{d} \sigma_{\mathrm{He}^{0}}^{+}, e t c\right)$ can be expressed (McGuire et al 1981) as a product of two separate parts:

$$
\mathrm{d} \sigma^{+}=Z_{\text {eff }}^{2}(Q) \mathrm{d} \sigma_{1}^{+} .
$$

The first part is an effective charge squared $Z_{\text {eff }}^{2}(Q)$ which is independent of the target; the second part, which depends on the target and is independent of the projectile, is the cross section $\mathrm{d} \sigma_{1}^{+}$for a bare projectile with $Z=1$. Here $Z_{\text {eff }}^{2}(Q)$ can be expressed as

$$
\left|Z_{\text {eff }}(Q)\right|^{2}=Z^{2}+2-4 Z\left|1+(Q / 2 Z)^{2}\right|^{-2}+2\left|1+(Q / 2 Z)^{2}\right|^{-4}
$$

for two projectile electrons (helium-like), as

$$
\left|Z_{\text {eff }}(Q)\right|^{2}=Z^{2}+1-2 Z\left|1+(Q / 2 Z)^{2}\right|^{-2}
$$

for one projectile electron (hydrogen-like), and as

$$
\left|Z_{\text {eff }}(Q)\right|^{2}=Z^{2}
$$

for 0 projectile electrons (bare nucleus), where $Z$ refers to the projectile charge. It is interesting to note that, at large $Q$, the antiscreening part of $Z_{\text {eff }}^{2}(Q)$ yields an incoherent limit $\left(Z^{2}+N\right)$ and total cross section is a sum of independent cross sections from the bare projectile $\left(\sim Z^{2}\right)$ and the $N$ electrons. Thus at large $Q$ this first Born ionisation cross section reduces to Bohr's free-collision model (Bohr 1948, Andersen et al 1987) where cross sections for the projectile nucleus and electrons simply add.

Now by using equations (1), (2), and the effective charge, the ratio of total double to total single ionisation cross sections can be written as

$$
\begin{aligned}
\mathscr{R} & =\iint Z_{\text {eff }}^{2}(Q) R(Q, \varepsilon) \frac{\mathrm{d} \sigma_{1}^{+}}{\mathrm{d} Q \mathrm{~d} \varepsilon} \mathrm{d} Q \mathrm{~d} \varepsilon\left(\iint Z_{\text {eff }}^{2}(Q) \frac{\mathrm{d} \sigma_{1}^{+}}{\mathrm{d} Q \mathrm{~d} \varepsilon} \mathrm{d} Q \mathrm{~d} \varepsilon\right)^{-1} \\
& =\iint Z_{\text {eff }}^{2}(Q) R(Q, \varepsilon) \frac{\mathrm{d} \sigma_{1}^{+}}{\mathrm{d} Q \mathrm{~d} \varepsilon} \mathrm{d} Q \mathrm{~d} \varepsilon\left(\sigma^{+}\right)^{-1}
\end{aligned}
$$


This central result makes it possible to study $\mathscr{R}$ for projectiles with electrons in the first Born approximation and sets up a connection with bare projectiles.

The first Born cross sections for single ionisation by bare projectiles in (3) and (6) are well known (McDowell and Coleman 1970, Rudd et al 1985). However, $R(Q, \varepsilon)$ which includes information about two correlated continuum electrons is not known. Nevertheless, recent experimental observations (Shah and Gilbody 1985, Andersen et al 1987, DuBois 1987, DuBois and Toburen 1988, Giese and Horsdal 1988, Kamber et al 1988, Dubois and Kover 1989) can be used to make some guesses about the form of $R$. First, let us consider a recent experiment (Kamber et al 1988) of double ionisation of helium by proton impact at energies of $3 \mathrm{MeV}$ and $6 \mathrm{MeV}$. It has been found that although $R$ depends on both variables, the $Q$ dependence can be regarded as weak compared with the $\varepsilon$ dependence. The $R$ values for protons and photons differ by about a factor of two while the corresponding $Q$ values differ by about 50 . Thus at sufficiently high velocities, one can use $R(Q, \varepsilon) \simeq R(\varepsilon)$ if $Q$ does not vary too much. Between $\mathrm{H}^{+}$and $\mathrm{H}^{0}$ we expect that $Q$ varies by only a factor of two or less, so that the variation of $R$ may be small. Using $R(Q, \varepsilon) \simeq R(\varepsilon)$ and equation (4), it is obvious that after integrating over $Q$, equations (3) and (6) will have a simple form, namely, $\mathscr{R} \simeq \int \mathrm{d} \varepsilon R(\varepsilon)\left(1 / \sigma^{+}\right) \mathrm{d} \sigma^{+} / \mathrm{d} \varepsilon$. Introducing a density distribution, $\rho(\varepsilon)=$ $\left(1 / \sigma^{+}\right) \mathrm{d} \sigma^{+} / \mathrm{d} \varepsilon$, we have

$$
\mathscr{R}=\int R(\varepsilon) \rho(\varepsilon) \mathrm{d} \varepsilon .
$$

This derivation is more general than that of McGuire (1984) where $R$ was taken as the photon ratio, $R_{\gamma}$, for all charged projectiles. Also our derivation is not restricted by the validity of the Bethe approximation.

By fitting experimental data, McGuire used a rather simple monotonic relation for $R(\varepsilon)$, namely

$$
R(\varepsilon)=\frac{\varepsilon}{\varepsilon_{1 / 2}+\varepsilon} R_{\infty}
$$

where $\varepsilon_{1 / 2}$ is a fitting parameter, $R_{\infty}$ is the limiting value of $R(\varepsilon)$ when $\varepsilon \rightarrow \infty$. This monotonic relation $R(\varepsilon)$ is shown in figure 1 (full curve), where $R_{\infty}$ is taken as $3 \%$.

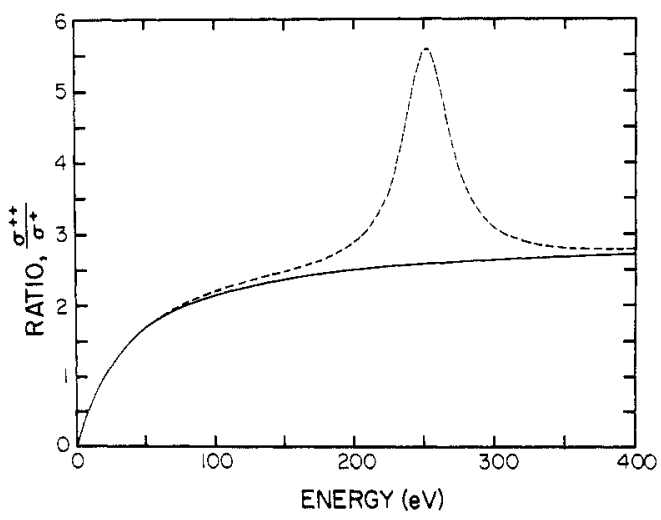

Figure 1. Phenomenological ratio (in \%) for differential double to differential single ionisation as a function of ejected electron energy (in eV). Full curve, monotonic ratio $R=\left[\varepsilon /\left(\varepsilon_{1 / 2}+\varepsilon\right)\right] R_{\infty}$, where $\varepsilon_{1 / 2}$ is taken as $40 \mathrm{eV}, R_{\infty}$ as $3 \%$. Broken curve, a peak is added to the monotonic ratio at $\varepsilon=250 \mathrm{eV}$ with a width of $20 \mathrm{eV}$. 
A qualitative understanding of $\mathscr{R}$ for projectiles with and without electrons can be given using (5) and (6) with $\rho(\varepsilon)=\left(1 / \sigma^{+}\right) \int_{Q_{\min }}^{\infty} Z_{\text {eff }}^{2}(Q)\left(\mathrm{d} \sigma_{1}^{+} / \mathrm{d} Q \mathrm{~d} \varepsilon\right) \mathrm{d} Q$. For the small $Q$ region, equations $(5 a)$ and $(5 b)$ give $\left|Z_{\text {eff }}(Q)\right|^{2}=(Z-N)^{2}$, which is zero for $Z=N$, whereas in $(5 c)$ it is $Z^{2}$. Thus, in this region where $\mathrm{d} \sigma_{1}^{+} / \mathrm{d} Q \mathrm{~d} \varepsilon$ is large, the contribution to the integral for bare projectiles will be larger than for projectiles with electrons. Because $Q_{\min }=(\varepsilon+I) / 2 v_{\mathrm{p}}$, where $I$ is the target ionisation potential (and therefore $I / 2 v_{\mathrm{p}}$ is small), small values of $Q$ correspond to small values of $\varepsilon$ so that enhancement of $\rho(\varepsilon)$ for bare projectiles relative to projectiles with electrons occurs for small $\varepsilon$.

The distribution $\rho(\varepsilon)$ is normalised to one. For projectiles with electrons, $\rho(\varepsilon)$ is small at small $\varepsilon$ and is therefore peaked at larger $\varepsilon$. And this is the same region of $\varepsilon$ in which $R(\varepsilon)$, from equation (7) and figure 1 , is appreciable. In contrast, for bare projectiles $\rho(\varepsilon)$ is peaked at small $\varepsilon$ where $R(\varepsilon)$ is small. Consequently $\mathscr{R}$ is greater for $\mathrm{H}^{0}$ than for $\mathrm{H}^{+}$. Our qualitative understanding is consistent with our quantitative calculation in table 1.

The ratio $\mathscr{R}$ is even bigger for $\mathrm{He}^{0}$ than for $\mathrm{H}^{0}$ since $Z_{\text {eff }}^{2}(Q)$ is twice as large in the small $Q$ region for $\mathrm{He}^{0}$ than for $\mathrm{H}^{0}$. This suggests that for $\mathrm{H}^{+}, \mathrm{H}^{0}$ and $\mathrm{He}^{0}$ :

$$
\mathscr{R}_{\mathbf{H}^{+}}<\mathscr{R}_{\mathbf{H}^{0}}<\mathscr{R}_{\mathrm{He}^{0}} \text {. }
$$

The monotonic relation assumed in (8) conflicts with recent studies by Giese and Horsdal (1988), Reading et al (1989) and Vegh (1989). Giese and Horsdal observed a peak in the ratio of double to single ionisation of He by proton impact as a function of scattering angle $\theta$ (i.e. $Q$ ), suggesting a possible non-monotonic $R(\varepsilon)$. We use the binary encounter model (Kamber et al 1988) to relate $\varepsilon$ and $Q$ to obtain a double binary encounter peak in $R(\varepsilon)$ consistent with the observations of Giese and Horsdal. We assume that the projectile has a binary encounter with each of the two electrons in helium. In figure 1 , a peak at $\varepsilon=250 \mathrm{eV}$ which corresponds to binary encounter electrons from $500 \mathrm{keV} \mathrm{amu}^{-1}$ projectiles is added to the monotonic curve. The width of the peak is taken as $20 \mathrm{eV}$, approximately corresponding to the width of the electron momentum distribution in helium. The height of $3 \%$ is taken to fit experiment.

In table 1 , we present calculations for $\mathscr{R}$ in two cases. The first (denoted as $\mathscr{R}^{1}$ ) is for the calculation using the monotonic expression in equation (8). The second (denoted as $\mathscr{R}^{2}$ ) is for the calculation using the monotonic relation together with a peak centred at $\varepsilon=250 \mathrm{eV}$ since a double binary collision could account for the peak observed by Giese and Horsdal. We have varied the position of the peak from $1 \mathrm{keV}$ (corresponding to binary encounter electrons from $2 \mathrm{MeV} \mathrm{amu}^{-1}$ projectiles) down to $40 \mathrm{eV}$. At $40 \mathrm{eV}$ the peak increases all the ratios by about a factor of two. As the peak moves to larger $\varepsilon$, its influence on $\mathscr{R}$ decreases. Although the various forms of $R(\varepsilon)$ give somewhat different values for $\mathscr{R}$, all of those we tried agree with (9).

In the development above, several points are worth further discussion. First, the expression for $Z_{\text {eff }}^{2}(Q)$ is derived using a closure approximation. We expect that the closure correction of Anholt (1986) to $(5 a)-(5 c)$ is small in our case since the collision velocity is well above the threshold velocity for ionisation by electrons sensitive to $Q$. For charged projectiles where contributions from small $Q$ and small $\varepsilon$ are significant in (6), $\mathscr{R}$ is somewhat sensitive to the difference in the single and double ionisation potential (McGuire 1984). However, for neutral projectiles $\mathscr{R}$ is not sensitive since $Q \approx Q_{\min }$ contributions are damped by $Z_{\text {eff }}^{2}(Q)$. Calculations confirmed that the ordering of the various ratios $\mathscr{R}$ for different projectiles does not depend on how ionisation potentials are used. Another interesting aspect is that the ratios obtained for the various projectiles are all smaller than either the differential $R_{\infty}$ limit of $3 \%$ or the observed 
Table 1. Ratio $(\mathscr{R})$ of total double to total single ionisation of helium $\mathrm{H}^{+}$and $\mathrm{H}^{0}$ impact at $1 \mathrm{MeV}, 2 \mathrm{MeV}$ and $5 \mathrm{MeV}$ and by $\mathrm{He}^{0}$ impact at $2 \mathrm{MeV} \mathrm{amu}^{-1}$ and $5 \mathrm{MeV} \mathrm{amu}{ }^{-1}, \sigma^{+}$ denotes the calculated first Born single ionisation cross section. $\mathscr{R}^{k}$ denotes the calculation using the monotonic $R ; \mathscr{R}^{2}$ denotes the calculation using the modified $R$ with a peak added at $\varepsilon=250 \mathrm{eV}$. At or below $1 \mathrm{MeV} \mathrm{amu}^{-1}, Z^{2}$ dependence may be altered by higher order terms in $Z$.

\begin{tabular}{llllll}
\hline Projectile & $\begin{array}{l}\text { Energy } \\
\left(\mathrm{MeV} \mathrm{amu}^{-1}\right)\end{array}$ & $\begin{array}{l}\sigma^{+} \\
\left(\pi a_{0}^{2}\right)\end{array}$ & $\begin{array}{l}\mathscr{R}^{\mathrm{i}} \\
(\%)\end{array}$ & $\begin{array}{l}\mathscr{R}^{2} \\
(\%)\end{array}$ & $\begin{array}{l}\text { Experiment } \\
(\%)\end{array}$ \\
\hline $\mathrm{H}^{+}$ & 1 & 0.073 & 0.35 & 0.41 & $0.33^{\mathrm{a}}$ \\
& 2 & 0.040 & 0.33 & 0.38 & $0.28^{\mathrm{a}}$ \\
& 5 & 0.018 & 0.31 & 0.35 & $0.26^{\mathrm{b}}$ \\
$\mathrm{H}^{0}$ & 1 & 0.065 & 0.69 & 0.80 & $0.83^{\mathrm{c}}$ \\
& 2 & 0.033 & 0.69 & 0.81 & - \\
& 5 & 0.013 & 0.69 & 0.81 & - \\
$\mathrm{He}^{0}$ & 2 & & & & - \\
& 5 & 0.041 & 1.04 & 1.23 & - \\
\hline
\end{tabular}

a Shah and Gilbody (1988).

b Andersen et al (1987).

${ }^{c}$ DuBois and Kover (1989).

photon ratio of $5 \%$. This implies that $\mathscr{R}_{\gamma}$ could set an upper limit on both $\mathscr{R}_{\infty}$ (which could differ for various projectiles) and the right-hand side of (9). Our results also imply inequalities for other systems, e.g. $\mathscr{R}_{\mathrm{H}^{+}}=\mathscr{R}_{\mathrm{He}^{2+}}<\mathscr{R}_{\mathrm{He}^{+}}<\mathscr{R}_{\mathrm{He}^{0}}$.

This work was supported by the Division of Chemical Sciences, Office of Energy Research, US Department of Energy.

\section{Reference}

Åberg T 1973 Proc. Int. Conf. on Inner Shell Ionisation Phenomena and Future Applications (Atlanta, GA) ed R W Finks et al (Oak Ridge, TN: US Atomic Energy Commission) p 1509

Andersen L H, Hvelplund P, Knudsen H, Moller S P, Sorensen A H, Elsener K, Rensfelt K-G and Uggerhoj E 1987 Phys. Rev. A 363612

Andersen L H, Nielsen L B and Sorensen J 1988 J. Phys. B: At. Mol. Opt. Phys. 21 1587-1602

Anholt R 1986 Phys. Lett. 114A 126

Bates D R and Griffing G W 1955 Proc. $R$. Soc. A 68831

Bell K L, Dose V and Kingston A E 1969 J. Phys, B: At. Mol. Phys. 2653

Bohr N 1948 K. Dansk. Vidensk. Selsk. Mat. Fys. Meddr. 18 No 8

Briggs J S and Taulbjerg K 1978 Topics in Current Physics vol 5 ed I Sellin (New York: Springer) p 105

Byron F W and Joachain C J 1967 Phys. Rev. 1641

Carlson T A 1967 Phys. Rev. 156142

Carlson T A and Nextor C W 1973 Phys. Rev. A 82887

Carter S L and Kelly H P 1981 Phys. Rev. A 24170

DuBois R D 1987 Phys. Rev. A 362585

DuBois R D and Kover A 1989 Phys. Rev. A 403605

DuBois R D and Toburen L H 1988 Phys. Rev. A 383960

Giese J P and Horsdal E 1988 Phys. Rev. Lett. 602018

Haugen H K, Andersen L H, Hvelplund P and Knudsen H 1982 Phys. Rev. A 261962

Inokuti M 1971 Rev. Mod. Phys. 43297

— 1979 Argonne National Laboratory Report No ANL-76-88 p 177 (unpublished) 
Kamber E Y, Cocke L, Cheng S and Varghese S L 1988 Phys. Rev. Lett. 602026

Manson S T, Toburen L H, Madison D H and Stolterfoht N 1975 Phys. Rev. 1260

McDowell M R C and Coleman J P 1970 Introduction to the Theory of Ion-Atom Collisions (Amsterdam:

North-Holland) ch 7 pp 337-9

McGuire J H 1984 J. Phys. B: At. Mol. Phys. 17 L779

- 1987 Phys. Rev. A 361174

McGuire J H, Stolterfoht N and Simony P R 1981 Phys. Rev. A 2497

Mittleman M H 1966 Phys. Rev. Lett. 16498

Quarles C A 1973 Proc. Int. Conf. on Inner Shell Ionisation Phenomena and Future Applications (Atlanta, GA) ed R W Finks et al (Oak Ridge, TN: US Atomic Energy Commission) p 1617

Reading J F, Ford A L and Fang X 1989 Phys. Rev. Lett. 62245

Rudd M E, Kim Y-K, Madison D H and Gallagher J W 1985 Rev. Mod. Phys. 57965

Shah M B and Gilbody H B 1985 J. Phys. B: At. Mol. Phys. 18899

Vegh L 1989 J. Phys. B: At. Mol. Opt. Phys. 22 L35 